\title{
Perfil da mortalidade de mulheres na região Nordeste do Brasil em decorrência de
}

\section{depressão}

\author{
Mortality profile of women in the Northeast region of Brazil due to depression \\ Perfil de mortalidad de las mujeres de la región Nordeste de Brasil por depresión
}

\author{
Maria Gabriela Pereira da Silva \\ ORCID: https://orcid.org/0000-0002-8815-0526 \\ Universidade Estadual da Paraíba, Brasil \\ E-mail: maria.gabriela.pereira@aluno.uepb.edu.br \\ Edwirde Luiz Silva Camêlo \\ ORCID: https://orcid.org/0000-0003-3686-927X \\ Universidade Estadual da Paraíba, Brasil \\ E-mail: edwirde@uepb.com \\ Dalila Camêlo Aguiar \\ ORCID: https://orcid.org/0000-0002-2901-4639 \\ Universidade de Granada, Espanha \\ E-mail: dalilacamelo@correo.ugr.es
}

\begin{abstract}
Resumo
Introdução: A depressão é um transtorno que afeta mais de 300 milhões de pessoas no mundo, contudo, o número de mulheres que apresentam esse transtorno é alarmante. Objetivo: Analisar o perfil de mulheres que morreram de alguma causa associada à depressão na região Nordeste do Brasil, entre 2014 a 2019. Métodos: Usamos uma tabela de contingência para apresentar um resumo das variáveis, matriz de correlação para identificação das relações e para reduzir a dimensionalidade sem perder informações e explicar essas variáveis em termos de suas componentes aplicamos a Análise de Componentes Principais. A coleta de dados foi realizada a partir dos dados do Sistema de Informação sobre Mortalidade (SIM), selecionando também a categoria do CID-10 que correspondem a episódios depressivos e transtorno depressivo recorrente (F32 e F33). As variáveis selecionadas foram raça, estado civil e escolaridade levando em consideração apenas pessoas do sexo feminino. Os dados foram tabulados no Excel e analisados no programa estatístico R versão 3.5.2. Resultados: Ocorreram 613 óbitos no sexo feminino nesse período, sendo principalmente mulheres pardas e casadas. As variáveis estão fortemente correlacionadas e hipóteses são levantadas: preconceito, vulnerabilidade social, falta de suporte social, relacionamento abusivo, mulheres procuram mais ajuda do que os homens. Conclusão: O perfil de mortalidade varia em função de raça e estado civil, sendo Bahia e Ceará os dois estados que obtiveram os índices mais significativos. Logo, se fazem necessárias políticas públicas, investimento na prevenção e promoção de qualidade de vida, rede de apoio a mulheres que passam por alguma situação que seja considerada fator de risco para um quadro depressivo.
\end{abstract}

Palavras-chave: Mortalidade; Saúde da mulher; Depressão.

\begin{abstract}
Introduction: Depression is a disorder that affects over 300 million people worldwide; however, the number of women who present this disorder is alarming. Objective: To analyze the profile of women who died from some cause associated with depression in the Northeast region of Brazil during 2014-2019. Methods: We used a contingency table to summarize the variables, a correlation matrix to identify the relationships, and Principal Component Analysis to reduce dimensionality without losing information and explain these variables in terms of their components. Data collection was carried out based on data from the Mortality Information System (SIM), selecting the ICD-10 category corresponding to depressive episodes and recurrent depressive disorder (F32 and F33). The variables selected were race, marital status, and education, taking into consideration only females. The data were tabulated in Excel and analyzed using the statistical program R version 3.5.2. Results: There were 613 female deaths during this period, mainly brown and married women. Variables are strongly correlated, and hypotheses are raised: prejudice, social vulnerability, lack of social support, abusive relationship, women seek more help than men. Conclusion: The mortality profile varies according to race and marital status, with Bahia and Ceará the two states that obtained the most effective rates. Therefore, public policies, investment in prevention and promotion of quality of life, a support network for women who go through a situation considered a risk factor for a depressive condition are necessary.
\end{abstract}

Keywords: Mortality; Women's health; Depression. 


\begin{abstract}
Resumen
Introducción: La depresión es un trastorno que afecta a más de 300 millones de personas en todo el mundo, sin embargo, la cantidad de mujeres que padecen este trastorno es alarmante. Objetivo: Analizar el perfil de mujeres fallecidas por alguna causa asociada a depresión en la región Nordeste de Brasil, entre 2014 y 2019. Métodos: Utilizamos una tabla de contingencia para presentar un resumen de las variables, una matriz de correlación para identificar las relaciones y reducir la dimensionalidad sin perder información y explicar estas variables en términos de sus componentes, aplicamos Análisis de Componentes Principales. La recogida de datos se realizó utilizando datos del Sistema de Información de Mortalidad (SIM), seleccionando también la categoría CIE-10 correspondiente a episodios depresivos y trastorno depresivo recurrente (F32 y F33). Las variables seleccionadas fueron raza, estado civil y educación teniendo en cuenta solo a las mujeres. Los datos se tabularon en Excel y se analizaron con el programa estadístico R versión 3.5.2. Resultados: Hubo 613 muertes de mujeres durante este período, principalmente mujeres morenas y casadas. Las variables están fuertemente correlacionadas y se plantean hipótesis: prejuicio, vulnerabilidad social, falta de apoyo social, relación abusiva, las mujeres buscan más ayuda que los hombres. Conclusión: El perfil de mortalidad varía en función de la raza y el estado civil, siendo Bahía y Ceará los estados que obtienen los índices más significativos. Por tanto, son necesarias las políticas públicas, la inversión en la prevención y la promoción de la calidad de vida, y una red de apoyo para las mujeres que pasan por una situación que se considera un factor de riesgo para la depresión.
\end{abstract}

Palabras clave: Mortalidad; Salud de la mujer; Depresión.

\title{
1. Introdução
}

O olhar perante a depressão normalmente é entendido pelo senso comum, de maneira equivocada e estereotipada, sendo postulada como apenas uma tristeza intensa e isso vem sendo modificado e ressignificado ao longo dos anos. Sob a ótica de Cordás e Emílio (2017), a diferença está na alteração do comportamento da pessoa, a tristeza só vai se configurar como depressão se afetar diretamente a qualidade de vida do indivíduo, comprometendo sua saúde e deixando afazeres de lado.

Nessa conjuntura, o DSM-5 intitula uma perspectiva mais detalhada onde a depressão é separada em níveis de acordo com a variação e quantificação dos sintomas. O humor obrigatoriamente deve ser um dos traços que sofre alguma mudança e pode ser "triste, vazio ou irritável, acompanhado de alterações somáticas e cognitivas que afetam significativamente a capacidade de funcionamento do indivíduo". Por outro lado, no CID-10 especifica-se também a quantidade de sintomas que se tem em cada nível, sendo: a) leve com 2 a 3 sintomas e ainda sendo capaz de realizar algumas atividades; b) moderado com quatro ou mais sintomas e o indivíduo começa a apresentar dificuldades nos afazeres; c) nível mais grave observa-se vários indícios somáticos, além de que ideias e atos suicidas são comuns.

Considerada pela Organização Pan-Americana da Saúde/Organização Mundial da Saúde (OPAS/OMS) a principal causa de incapacidade no mundo, a depressão é uma doença que envolve vários fatores de risco, como a influência genética atuando com fatores sociais, culturais e outros. Além disso, mais de 300 milhões de pessoas são diagnosticadas com depressão e, quando considerado os casos mais graves, podem ter atos e pensamentos suicidas. É constatado que cerca de 800 mil pessoas morrem por suicídio a cada ano - sendo essa a segunda principal causa de morte entre jovens. Apesar de afetar muitas pessoas, a OPAS/OMS salienta que, em alguns países há um percentual de menos de 10\% de tratamentos eficazes, o que corrobora para esse panorama é a falta de recursos, a falta de profissionais treinados e o estigma social associado aos transtornos mentais. Segundo esse mesmo órgão, nos próximos 20 anos a depressão será, possivelmente, uma das principais causas de doenças no mundo, o que acarreta prejuízos na qualidade de vida, no trabalho e nos estudos.

Sob outra perspectiva, o cenário no Brasil não é diferente, 1.049 pessoas morreram no Nordeste entre 2014 e 2019 em decorrência de episódios depressivos e transtorno depressivo recorrente, sendo 613 mulheres. A Bahia lidera os casos no nordeste brasileiro com 306 mortes e o Ceará com 208 pessoas, sendo o $2^{\circ}$ e o $6^{\circ}$ estado no Brasil, respectivamente, com o maior índice de mortes associadas à depressão, conforme informações do Sistema Datasus do Ministério da Saúde.

Baptista e Oliveira (1999) referem em seu estudo que a mulher sente de maneira mais direta que o homem, procuram mais ajuda em serviços sociais, já que culturalmente os homens não fazem isso, ao invés de irem em busca de ajuda, procuram estratégias de enfrentamento como a bebida. Na perspectiva do fator genético, desde a puberdade a mulher lida com uma explosão 
de hormônios, que inclui o estrógeno e a progesterona. O estrógeno é um hormônio que sempre que sua produção cai, sentimentos como tristeza, cansaço, irritação e preocupação aparecem. Ele pode cessar na TPM, mas no período pós-parto também é comum, o que pode ocasionar a depressão pós-parto, podendo estar relacionado a outras variáveis como o casamento, gravidez indesejada e falta de suporte. Somado a tudo isso, socialmente a mulher tem múltiplos papéis a desempenhar: esposa, dona de casa, mãe/avó, filha, às vezes, trabalhadora (Caixeta e Barbato, 2004). Trazendo à tona o sentimento de infelicidade e exaustão, o que pode colaborar para um quadro depressivo.

A fim de compreender sua correlação com o sexo, evidencia-se como essa demanda da saúde pública precisa ser mais estudada, uma vez que as mulheres têm duas vezes mais probabilidade do que os homens de sofrer de depressão, isto porque existem diversos fatores que podem aumentar o risco de desenvolvimento da doença (Kuehner, 2016). O estudo em questão busca cruzar, entre si, dados do Sistema de Informações de Mortalidade (SIM) em um período de anos favorável conforme a disponibilidade de dados e analisar o perfil de mortalidade de mulheres nos estados do Nordeste em decorrência de depressão. Através dessa análise busca-se pontuar a necessidade de se aprofundar na investigação desses casos procurando observar o perfil de mulheres afetadas.

\section{Metodologia}

Neste trabalho foi realizado um estudo descritivo e quantitativo que segundo Pereira A. S. et al. (2018) consiste na coleta de dados e posteriormente com a ajuda de métodos matemáticos ocorre a sua análise, é uma característica de estudos estatísticos a preocupação com números porque é a partir deles que consegue-se estipular algumas informações, no caso o perfil de mulheres.

A coleta de dados foi realizada utilizando o site do Sistema de informações e mortalidade (SIM), a fim de investigar o perfil de mulheres que vieram a óbito em decorrência de episódios depressivos e transtornos depressivos recorrentes. Foi feito o cruzamento de dados como raça, escolaridade, estado civil e faixa etária (por região e unidade de federação) com o sexo feminino no período de 2014 a 2019 na região Nordeste.

Uma vez efetuada a coleta de dados, foram organizados em planilhas através do Microsoft Excel 365 na versão do Windows 10, posteriormente, foi analisado eletronicamente com o auxílio do programa estatístico R versão 3.5.2. Analiticamente utilizou-se uma tabela de contingência; matriz de correlação na identificação das relações entre as variáveis e para simplificar a complexidade de espaços amostrais com muitas dimensões, ao mesmo tempo em que preserva sua informação recorremos a Análise de Componentes Principais (ACP). Entretanto, como o objetivo do ACP é reduzir a dimensionalidade, muitas vezes é interessante usar o número mínimo de componentes que são suficientes para explicar os dados. Para isto, temos os gráficos scree plot que aparece de maneira descendente e isso acontece, pois, os primeiros componentes serão responsáveis por mostrar aquele que tiveram maior variação e o mapa de fatores que mostra o padrão que existe dentro dos dados, trata-se de um mapa perceptual que os representa em um espaço bidimensional.

\section{Resultados e Discussão}

Na Figura 1, temos os dados representados numa tabela de contingência contendo dez variáveis (colunas) e nove linhas com os respectivos valores das variáveis em relação a cada um dos sete estados da região Nordeste. Os valores maiores são apresentados por um destaque na cor azul. 
Figura 1 - Tabela de contingência entre as variáveis e estados.

\begin{tabular}{|c|c|c|c|c|c|c|c|c|c|c|}
\hline & Vievas & Casadas & Soteiras & Separados & Branca & Parda & Preta & Ers_Medo & Ens Fund & Feminino \\
\hline MA & 6 & 13 & 11 & 1 & 6 & 24 & 6 & 1 & 15 & 36 \\
\hline PI & 8 & 8 & 12 & 1 & 10 & 16 & 4 & 1 & 16 & 31 \\
\hline $\mathrm{CE}$ & 47 & 41 & 23 & 2 & 37 & 30 & 5 & 6 & 59 & 124 \\
\hline$R N$ & 8 & 11 & 9 & 1 & 14 & 14 & 1 & 1 & 15 & 32 \\
\hline PB & 11 & 15 & 6 & 4 & 16 & 26 & 3 & 1 & 14 & 46 \\
\hline $\mathrm{PE}$ & 17 & 25 & 17 & 3 & 23 & 39 & 3 & 5 & 32 & 67 \\
\hline AL. & 10 & 12 & 7 & 1 & 12 & 21 & 1 & 1 & 9 & 33 \\
\hline SE & 14 & 32 & 8 & 3 & 21 & 39 & 4 & 1 & 32 & $A B$ \\
\hline BA & 45 & 62 & 40 & 5 & 50 & 89 & 19 & 4 & 68 & 176 \\
\hline
\end{tabular}

Fonte: Autores (2021).

Durante o período de 2014 a 2019 no Nordeste do Brasil 613 mulheres vieram a óbito devido alguma causa associada à depressão. Dentre elas, predominantemente eram mulheres de cor parda $(348 ; 56,8 \%)$, casadas $(219 ; 35,7 \%)$ e tinham oito anos de escolaridade $(260 ; 42,4 \%)$.

É notório o índice alarmante da Bahia $(176 ; 28,7 \%)$ e do Ceará $(124 ; 20,2 \%)$, onde respectivamente os números são bem maiores. Já os estados do Maranhão (MA), Piauí (PI), Rio Grande do Norte (RN) e Alagoas (AL) apresentaram as menores ocorrências de mortalidade.

No ano de 2020, período em que a pandemia do Covid-19 atingiu seu ápice, a Bahia passou a receber mais de 8 milhões de reais para ampliar o suporte da saúde mental da população, enquanto o Ceará em $2^{\circ}$ lugar recebeu 4,8 milhões. Logo, é uma política pública que não se tinha antes apesar da necessidade alarmante, mas que foi ainda mais preocupante pelo histórico e devido ao período pandêmico onde é esperado um aumento de casos de doenças e transtornos mentais (Brasil, 2020).

Segundo o representante da Coordenação de Disseminação de Informações do IBGE na Bahia, Joilson Souza (2015), que colocou em pauta que homens têm muita dificuldade em buscar ajuda e por isso o número de mulheres é quase o dobro dos homens. Nessa linha de raciocínio, pacientes estigmatizados internalizam essas visões estigmatizantes e discriminatórias das pessoas em geral, dando origem ao chamado autoestigma. Há comprometimento da autoestima, mais incapacitação e menos resistência ao estresse (Rocha, Hara \& Paprocki, 2015), o que significa que o indivíduo que se encontra, muitas vezes, em uma esfera com muito preconceito a respeito da doença, se inibindo de procurar ajuda.

Observa-se que as mulheres pardas e casadas (exceto Piauí) tiveram maiores ocorrências nos estados. A priori, é necessário entender sobre as mulheres consideradas pardas, com base em Lauro Gomes (2019), a pessoa que é considerada pardo, desde a infância, é colocado como eufemismos para "negro" ou "indígena" [...] e os eufemismos negam a essa pessoa parda a sua própria identidade, e os outros termos que o identificam acabam o sexualizando e o desumanizando, e isso ocorre principalmente com as mulheres. Não apenas a denominação da raça evidencia pelo que eles passam, mas ainda de acordo com dados do IBGE que correlacionam a desigualdade social por cor ou raça, no que desrespeito ao mercado de trabalho, apenas $29,9 \%$ são ocupados por pessoas pardas ou pretas, sendo que mulheres pretas ou pardas ainda recebem menos da metade do que os homens brancos. Na taxa de analfabetismo, pessoas negras e pardas também são maioria chegando até $9,1 \%$ enquanto brancos estão com uma taxa de 3,9\%, quando se trata de representação política isso também acontece, deixando $24,4 \%$ de pessoas pretas 
ou pardas com representação política. Nesse sentido, é constatado que mulheres pardas (que na verdade, possivelmente, são mulheres com um subtom mais claro, mas que não deixam de ser negras) sofrem com a desvantagem em muitos âmbitos, sendo causa de sofrimento mental. As consequências das desigualdades sociais incluem a carga desproporcional de responsabilidade imposta às mulheres, uma vez que muitas vezes elas carregam a carga adicional e desigual de responsabilidades domésticas e o estresse dos empregos de linha de frente (por exemplo, enfermeiras, trabalhadores de mercearia). Da mesma forma, as populações vulneráveis também correm o risco de maior exposição ao racismo e ao estigma, o que agrava ainda mais o sofrimento mental, aumentando a exclusão social e o isolamento (Fiocruz, 2020). Logo, o racismo, a insegurança financeira, desigualdade de gênero em casa e no mercado de trabalho, o estresse ocasionado, são eventos vitais para o desenvolvimento de uma depressão e seu agravamento.

Ao estudar a relação entre gênero e saúde mental, Santos evidencia a partir de pesquisas que o casamento e a maternidade antecedem o adoecimento psíquico entre as mulheres que pertencem à geração mais velha e às camadas populares com baixa escolaridade. A violência de gênero esteve presente nessas narrativas e ocupa um lugar central (Santos, 2009), sob a ótica desse mesmo autor é salientado que mesmo a mulher estando muitas vezes doente, ela ainda é incumbida de cuidar de tudo e de todos pois é um papel social imposto a ela. Há estudos indicando que mulheres que se encontram dentro de um relacionamento abusivo apresentam com maior frequência problemas de saúde física e mental, vários relacionamentos e maior possibilidade de ter companheiros que as impeçam de ter atividades relacionadas ao trabalho e estudo (Wilhelm \& Tonet, 2007). Quando falamos em relação abusiva, ainda surge o pensamento do porque as mulheres simplesmente não se afastam dos seus parceiros para evitar sofrimento maior ou porque não pedem ajuda, segundo Soares (2005), a mulher tem vergonha de pedir ajuda a alguém, além de que quando se trata de relacionamento abusivo a vítima já está isolada da sua rede de apoio, para ela o homem irá mudar e aquilo e é só uma fase, algumas mulheres ainda dependem financeiramente do cônjuge, o que dificulta com que ela se afaste da situação. Diante desse encadeamento, observa-se que os dados corroboram com outros estudos vinculados confirmando o que já havia sido constatado.

Com intuito de entender as relações entre as variáveis, ou seja, compreender a associação estatística no tocante ao grau pelo qual um par de variáveis estão linearmente relacionadas temos abaixo a matriz de correlação representada pela Figura 2.

Figura 2 - Matriz de correlação entre os valores das variáveis de mulheres coletadas no sistema de mortalidade.

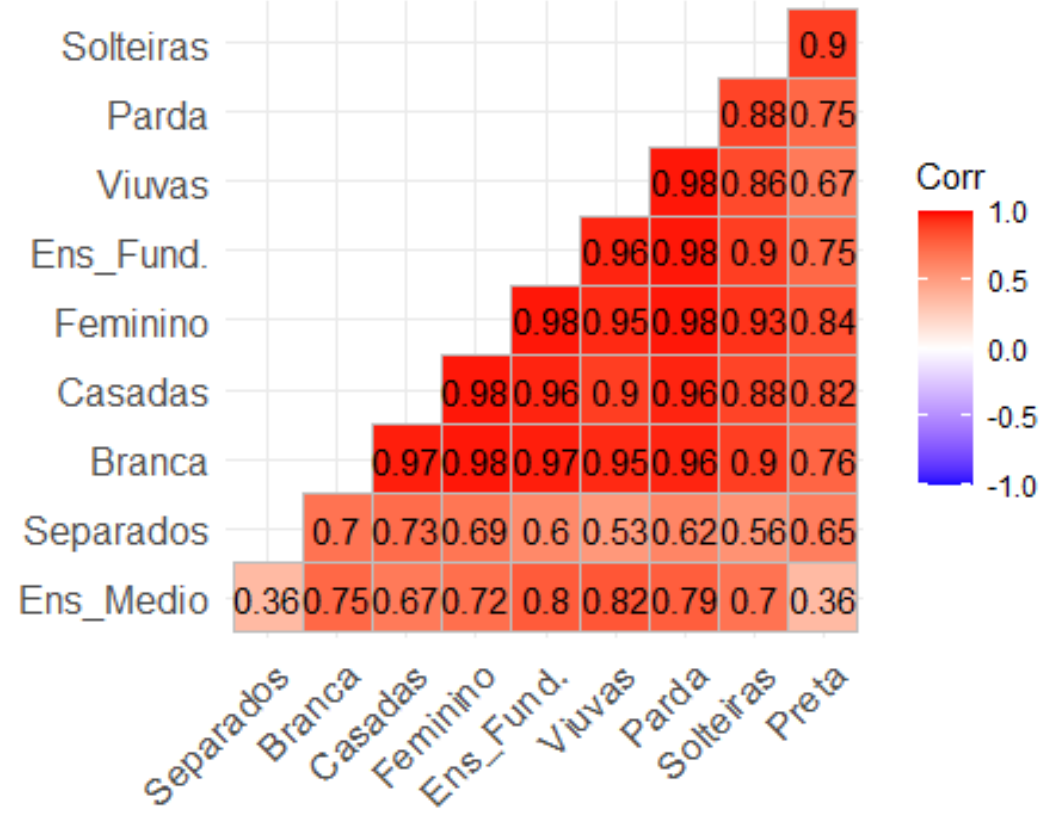

Fonte: Autores (2021). 
De acordo com a Figura 2, observam-se fortes correlações entre as variáveis, a menor correlação ocorreu entre as variáveis Ensino Médio com Separados $(0,36)$ e com cor Preta $(0,36)$. Apresentando uma das maiores correlações do gráfico a variável pardas têm um alto índice com a variável ensino fundamental, desconsiderando o feminino. Estudos de Dell'Aglio et al. (2004) constatou que a depressão e o desempenho em adolescentes em âmbito escolar apresentaram uma média maior entre as meninas levando em consideração também a faixa etária, moradia e sexo, onde foi constatado uma correlação com o sexo e com a séria em que estava. Algumas hipóteses foram levantadas, entre elas é a de que a criança pode estar inserida em um ambiente com abuso sexual, violência doméstica e negligência. Nele também foi percebido que a família desempenha um papel importante como suporte para a criança/adolescente. Segundo uma pesquisa de Campos et al. (2014) realizada em três escolas públicas, para surgir ou manter um quadro depressivo é preciso haver vários fatores correlacionados, tanto sociais, psicológicos e biológicos. No estudo, por exemplo, o histórico familiar, viver em famílias desestruturadas, estresse, sem suporte social, ser mulher, são variáveis que levam o indivíduo a tristeza e desinteresse, podendo agravar ou não.

Uma forma muito difundida de proceder na identificação do número ideal de componentes principais a serem utilizadas consiste em avaliar o percentual da variância explicada acumulada e selecionar o número mínimo de componentes além dos quais o aumento não é mais substancial, conforme observamos na Figura 3.

Figura 3 - Scree plot explicando a variação total dos dados nas dimensões.

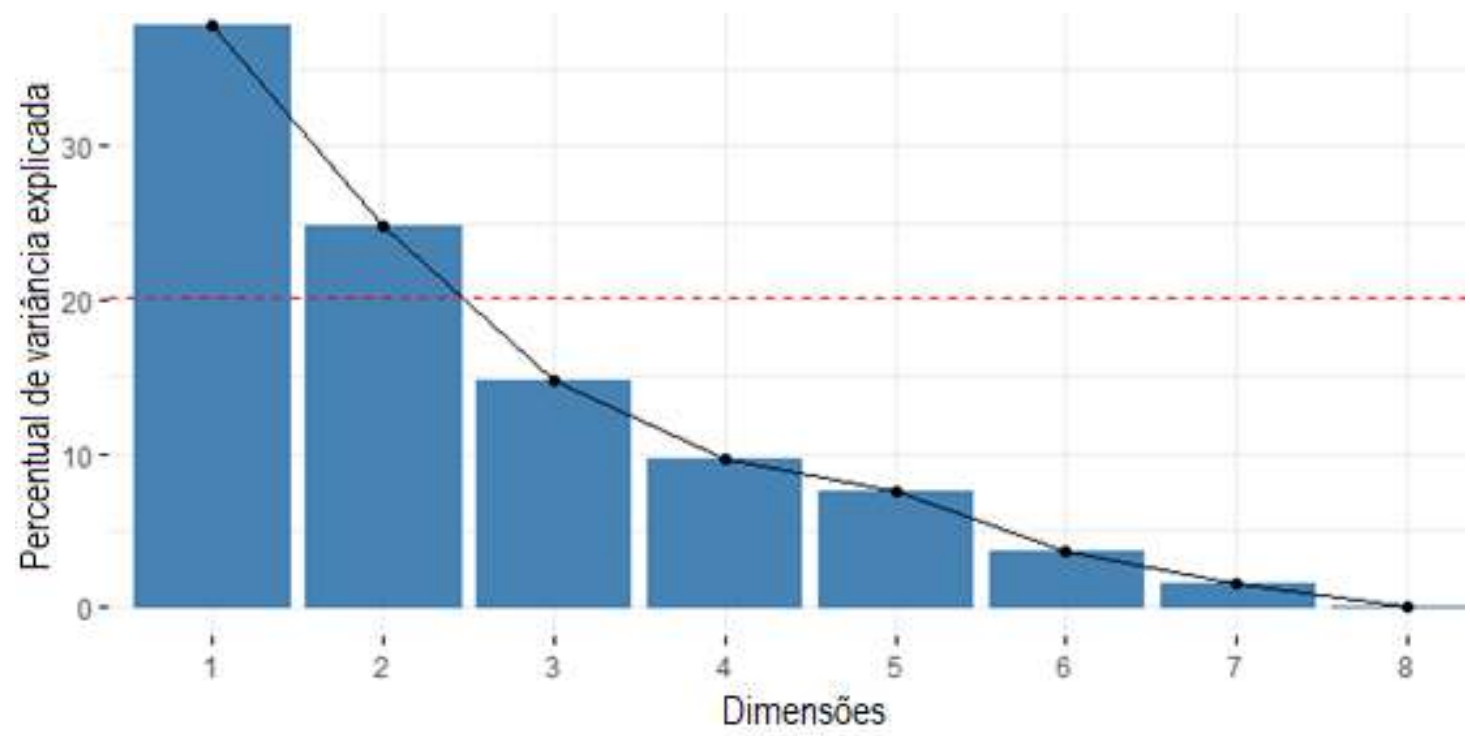

Fonte: Autores (2021).

Na figura 3, observa-se que há uma variabilidade maior na dimensão 1 e 2, sendo elas suficientes para descrição dos dados. Analisando de acordo com a tabela, a ACP mostra que CP1 explica 37.9\% da variância, enquanto CP2 explica 24.8\%. De acordo com o gráfico do scree plot, apenas as dimensões 1 e 2 devem ser utilizadas na solução. A dimensão 3 explica apenas $11,4 \%$ da inércia total, que está abaixo do autovalor médio $(14,71 \%)$ e é muito pequena para ser mantida na análise.

A representação bidimensional das duas primeiras componentes é observada na Figura 4. 
Figura 4 - Configuração espacial das variáveis associadas.

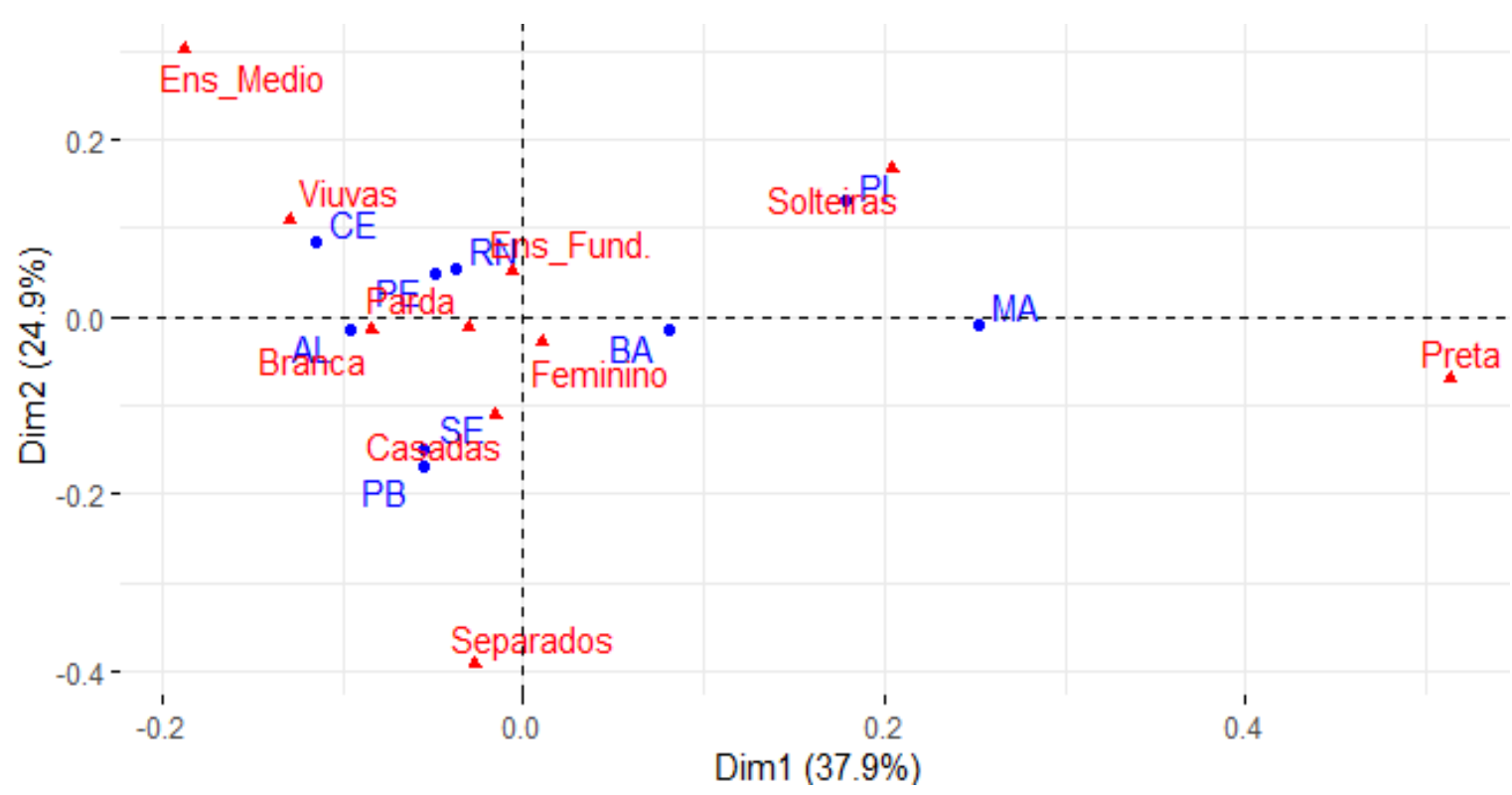

Fonte:

Autores (2021).

Na Figura 4, observa-se um mapa de fatores que possui 4 quadrantes, começando pelas que se encontram afastadas do centroide e próximas entre si como Solteiras e PI, Casadas na PB e SE, viúvas no CE, Brancas em AL, pardas em PE, por exemplo, são variáveis que têm um nível maior de associação. Separadas, pretas e ensino médio são as mais distantes do ponto central, não apresentando correlação com outras variáveis diretamente, mas que por si só demonstram alta variabilidade, é importante destacar que não apresentam entre si correlação, uma vez que estão em quadrantes opostos. Maranhão também se destaca na mortalidade em decorrência da depressão e isso pode estar relacionado com taxa de empregabilidade, violência, status social e ainda segundo estudos de Silva et al. sobre mulheres climatéricas no Maranhão, há uma prevalência de 34,4\% com depressão (superior em estudos mais antigos) o que pode ser um fator contribuinte.

\section{Conclusão}

No decorrer deste estudo observou-se que o perfil de mulheres que mais morreram em decorrência de uma depressão nos períodos estudados foram mulheres marcadas pela questão racial e estado civil, isso se dá por uma combinação de fatores, onde encontra-se incluso questão de opressão da mulher no geral, que aborda a objetificação e desumanização do ser, o que se torna mais acentuado quando se trata de mulheres pardas. Também fica marcado os fatores de relacionamento abusivo que isolam a mulher do suporte social, há violência (verbal ou física), e é um ciclo vicioso onde ela não consegue escapar dando margem para um sofrimento mental que pode evoluir para uma depressão.

Também houve uma forte correlação entre as variáveis, mas uma menor correlação entre, por exemplo, ensino fundamental e raça, isso se explica pela questão de que além de ser minoria nesses espaços, a questão sócio econômico impossibilita a busca pelo diagnóstico e suporte profissional, algo que também pode ser considerado é que mulheres pardas são, em sua maioria, mulheres negras com o subtom mais claro e assim sendo, tem um alto índice de mortalidade em decorrência do transtorno. Os casos estavam mais concentrados em duas principais regiões, sendo elas: Bahia e Ceará. Durante a elaboração desse estudo encontrou-se limitações em função dos dados que não foram quantificados porque estavam classificados como "ignorados" no site do Datasus.

Diante disso, sugere-se que novas pesquisas sejam realizadas a fim de compreender tal fenômeno de forma mais 
abrangente e profunda possibilitando a descoberta de novos perfis e correlações de óbitos de mulheres em decorrência de um quadro depressivo, sugere-se também pesquisas voltadas ao contexto pandêmico e como esses perfis mudaram ao longo desse período em um panorama não apenas regional, mas nacional.

\section{Referências}

American Psychiatric Association. (2014). DSM-5: Manual diagnóstico e estatístico de transtornos mentais. Artmed Editora.

Baptista, M. N., Baptista, A. S. D., \& Oliveira, M. D. G. D. (1999). Depressão e gênero: por que as mulheres deprimem mais que os homens?. Temas em psicologia, 7(2), 143-156.

Caixeta, J. E., \& Barbato, S. (2004). Identidade feminina: um conceito complexo. Paidéia (Ribeirão Preto), 14, 211-220.

Campos, J. R., Del Prette, A., \& Del Prette, Z. A. P. (2014). Depresión en la adolescencia: habilidades sociales y variables sociodemográficas como factores de riesgo/protección. Estudos e Pesquisas em Psicologia, 14(2), 408-428.

Capela, M. V., \& Capela, J. M. (2011). Elaboração de gráficos box-plot em planilhas de cálculo. In congresso de matemática aplicada e computacional da região sudeste-cnmac Sudeste (Vol. 1).

Cordás, T. A., \& Emilio, M. S. (2017). História da melancolia. Artmed Editora.

Dell'Aglio, D. D., \& Hutz, C. S. (2004). Depressão e desempenho escolar em crianças e adolescentes institucionalizados. Psicologia: Reflexão e Crítica, 17, 351-357.

Departamento de informática do sus - datasus. Informações de Saúde, Mortalidade: banco de dados.

Fortin, M. F., Côté, J., \& Filion, F. (2009). Fundamentos e etapas do processo de investigação.

Fundação Oswaldo Cruz: uma instituição a serviço da vida (2020). Desigualdades sociais provocam aumento do sofrimento mental em meio à pandemia da covid-19 [Internet]. https://www.cee.fiocruz.br/?q=desigualdades-sociais-provocam-aumento-do-sofrimento-mental-em-meio-a-pandemia-da-covid-1

Gomes, L. F. E. (2019). Ser Pardo: o limbo identitário-racial brasileiro e a reivindicação da identidade. Cadernos de Gênero e Diversidade, 5(1), 66-78.

Kuehner, C. (2017). Why is depression more common among women than among men? The Lancet Psychiatry, 4(2), 146-158.

Lin, S. C., Tyus, N., Maloney, M., Ohri, B., \& Sripipatana, A. (2020). Mental health status among women of reproductive age from underserved communities in the United States and the associations between depression and physical health. A cross-sectional study. Plos one, 15(4), e0231243. PLoS ONE 15(4): e0231243, 2020. https://doi.org/10.1371/journal.pone.0231243

Loesch, C., \& Hoeltgebaum, M. (2017). Métodos estatísticos multivariados. Saraiva Educação SA. São Paulo: Saraiva, 2012.

Ministério Da Saúde. Depressão: causas, sintomas, tratamentos, diagnóstico e prevenção. https://antigo.saude.gov.br/saude-de-a-z/depressao.

Ministério da Saúde. Saúde e Vigilância Sanitária https://www.gov.br/saude/pt-br/assuntos/noticias/bahia-recebe-mais-de-r-8-milhoes-para-ampliaratendimentos-em-saude-mental-no-sus.

Ministério da Saúde. Saúde e Vigilância Sanitária. https://www.gov.br/saude/pt-br/assuntos/noticias/ceara-recebe-mais-de-r-4-8-milhoes-para-ampliaratendimentos-em-saude-mental-no-sus.

Organização Mundial da Saúde. (1994). CID-10: Classificação Estatística Internacional de Doenças com disquete Vol. 1. Edusp.

Organização Pan-Americana da Saúde, Organização Mundial da Saúde para as Américas. Artigo. Depressão.https://www.paho.org/pt/topicos/depressao.

Paradis, E., \& Schliep, K. (2019). ape 5.0: um ambiente para a filogenética moderna e análises evolutivas em R. Bioinformatics , 35 (3), 526-528.

Pereira A. S. et al. (2018). Metodologia da pesquisa científica. [free e-book]. Santa Maria/RS. Ed. UAB/NTE/UFSM

R Core Team. Manual. R: A Language and Environment for Statistical Computing. R Foundation for Statistical Computing. Austria: Vienna, 2014. http://www.R-project.org/

Ramos, E. Estatística: poderosa ciência ao alcance de todos. website: http://www. ufpa. br/beiradorio/arquivo. Beira21/opini ao. html.

Santos, A. M. C. C. D. (2009). Articular saúde mental e relações de gênero: dar voz aos sujeitos silenciados. Ciência \& Saúde Coletiva, 14, $1177-1182$.

Silva, M. N. M. D., Brito, L. M. O., Chein, M. B. D. C., Brito, L. G. O., \& Navarro, P. A. D. A. S. (2008). Depressão em mulheres climatéricas: análise de mulheres atendidas ambulatorialmente em um hospital universitário no Maranhão. Revista de Psiquiatria do Rio Grande do Sul, 30, 150-154.Soares, M. B.Enfrentando a violência contra a mulher. Brasília: Secretaria Especial de Políticas para as Mulheres, 2005.

União dos Municípios da Bahia. (2015). Bahia tem alto índice de pessoas com depressão [Internet]. http://www.upb.org.br/noticias/bahia-tem-alto-indice-depessoas-com-depressao-aponta-ibge

Wickham, H., Averick, M., Bryan, J., Chang, W., McGowan, L. D. A., François, R., ... \& Yutani, H. (2019). Welcome to the Tidyverse. Journal of open source software, 4(43), 1686, https://doi.org/10.21105/joss.01686

Wilhelm, F. A., \& Tonet, J. (2017). Percepção sobre a violência doméstica na perspectiva de mulheres vitimadas. Psicologia Argumento, 25(51), 401-412. 